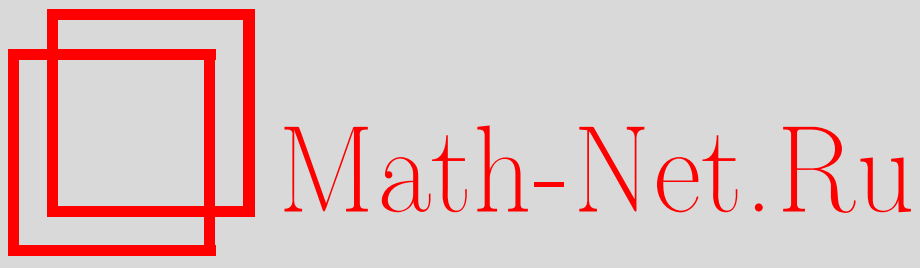

М. Г. Шур, Предельные теоремы для отношений, ассоциированных с самосопряженными операторами и симметричными цепями Маркова, Теория вероятн. и ее примен., 2000, том 45, выпуск 2, 268-288

DOI: https://doi.org/10.4213/tvp463

Использование Общероссийского математического портала Math-Net.Ru подразумевает, что вы прочитали и согласны с пользовательским соглашением http://www.mathnet.ru/rus/agreement

Параметры загрузки:

IP : 54.196 .121 .252

26 апреля 2023 г., 12:59:12

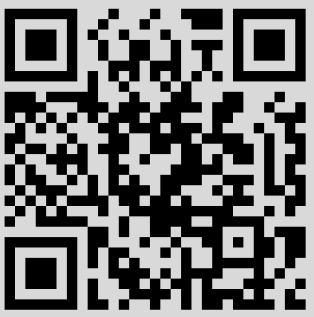




\title{
ПРЕДЕЛЬНЫЕ ТЕОРЕМЫ ДЛЯ ОТНОШЕНИЙ, АССОЩИИРОВАННЫХ С САМОСОПРЯЖЕННЫМИ ОПЕРАТОРАМИ И СИММЕТРИЧНЫМИ ЦЕПЯМИ MAPKOBA ${ }^{1)}$
}

\begin{abstract}
Получена простейшая предельная теорема для отношений, ассоциированных с самосопряженными операторами в пространствах типа $L_{2}$, оставляющими инвариантным конус неотрицательных элементов. C ее помощью доказаны предельные теоремы для отношений, отвечаюших симметричным цепям Маркова и симметричным ядрам в измеримых пространствах. В частности, для возвратных по Харрису симметричных цепей Маркова оказывается справедливым результат типа известной теоремы Ори (1961 г.) о дискретных возвратных симметричных цепях. Аналогичным образом обстоит дело и с неотрицательными симметричными квазифеллеровскими ядрами на локально компактных пространствах, являющимися лиувиллевыми в подходящем смысле.
\end{abstract}

Клюиевые слова и фразы: предельная теорема для отношений, самосопряженный оператор, возвратная по Харрису цепь Маркова, симметричное ядро, квазифеллеровское ядро, лиувиллево ядро.

Обычно предельные теоремы для отношений, ассоциированных с марковскими цепями на измеримых пространствах состояний, включают в себя или чрезмерные, или, несмотря на близость к необходимым, трудно проверяемые требования [2], [4], [10], [13]. Например, добротная теорема 7.4 из [2] содержит некое условие (7.6), сформулированное в терминах вложенных дискретных плотностей восстановления, проверка которого иной раз не менее трудна, чем доказательство основного утверждения, [10]-[13].

С другой стороны, в изящной теореме Ори [4], относящейся к дискретным симметричным цепям Маркова, равно как и в некоторых других ситуациях [10]-[14], подобные условия отсутствуют. К тому же сходные результаты удается получить и для невозвратных цепей Маркова,

\footnotetext{
"Московский тосударственный институт электроники и математики, Б. Трехсвятительский пер., 3/12, 109028 Москва, Россия.

1) Работа выполнена при поддержке Российского фонда фундаментальных исследований, проект № 96-01-01438.
} 
подчиненных условию лиувиллевости в подходяшей форме. Соответствующие примеры см. в $[10],[11],[13]$, но, вероятно, самый известный из них доставляется простыми $d$-мерными случайными блужданиями при $d \geqslant 3$ [14]. В связи с последним примером упомянем и теоремы типа Аве об асимптотической равнораспределенности симметричных блужданий на широком классе групп [12].

Основная цель нашей работы состоит в получении теорем типа Ори для симметричных возвратных по Харрису цепей Маркова и симметричных квазифеллеровских топологически лиувиллевых ядер (см. далее теоремы 2 и 3). Попутно получены и другие результаты (например, в предложении 1 дана новая характеризация параметра сходимости симметричного ядра). Предлагаемый подход базируется на новой простейшей предельной теореме для отношений, порожденных некоторыми самосопряженными операторами (см. теорему 1), и наша методика развивает использованную в $[10],[13]$.

Основная терминология и формулировки главных результатов отражены в п. 1 ; теоремы 1,2 и 3 доказаны в пп. 2, 4, 5 соответственно; в п. 3 , в основном, приведен вспомогательный материал; п. 6 , стоящий несколько особняком, дополняет теорему 3.

1. Терминология и основные результаты. Отправной точкой служат следующие соображения. Пусть $T$ - линейный ограниченный самосопряженный оператор в вещественном или комплексном гильбертовом пространстве $H$. Для $f \in H$ неравенство Коши-Буняковского дает:

$$
\left\|T^{n} f\right\|^{2}=\left(T^{n+1} f, T^{n-1} f\right) \leqslant\left\|T^{n+1} f\right\| \cdot\left\|T^{n-1} f\right\|, \quad n \geqslant 1
$$

Поэтому $\left\|T^{n} f\right\| \neq 0$ при $n \geqslant 1$ и последовательность $\left\{\left\|T^{n+1} f\right\| \cdot\left\|T^{n} f\right\|^{-1}\right.$; $n \geqslant 0\}$ не убывает, если $\|T f\| \neq 0$ (этот факт установлен в [3] с применением спектральной теории операторов). Таким образом, в случае $\|T f\| \neq 0$ можно положить

$$
\rho(f) \equiv \rho_{T}(f)=\lim _{n \rightarrow \infty}\left[\left\|T^{n+1} f\right\| \cdot\left\|T^{n} f\right\|^{-1}\right]=\lim _{n \rightarrow \infty}\left[\left\|T^{n} f\right\|^{1 / n}\right] \leqslant\|T\|,
$$

причем согласно последнему равенству

$$
\rho\left(T^{m} f\right)=\rho(f), \quad m \geqslant 0 .
$$

Кроме того, попутно выяснилось, что в том же случае

$$
\|T f\| \cdot\|f\|^{-1} \leqslant\left\|T^{n+1} f\right\| \cdot\left\|T^{n} f\right\|^{-1} \leqslant \rho(f) \leqslant\|T\|, \quad n \geqslant 0 .
$$

Если же $\|T f\|=0$, то удобно считать $\rho(f)=0$. 
Всюду в дальнейшем рассматриваются измеримое пространство $(E, \mathscr{B})$ и $\sigma$-конечная мера $\xi$ на $\mathscr{B}$. В роли $H$ будет фигурировать вешественное пространство $L_{2}=L_{2}(\xi)$, наделенное стандартным скалярным произведением $(\cdot, \cdot)$. Поскольку $f, g \in L_{2}$ являются классами эквивалентности $\mathscr{B}$-измеримых функций с суммируемыми по $\xi$ квадратами, неравенства типа $f \geqslant g$, где $f, g \in L_{2}$, будут интерпретироваться как их аналоги для представителей этих классов, выполняющиеся п.в. (т.е. $\xi$-почти всюду). С учетом сказанного определяются как элемент $f \vee g=\max (f, g)$ для тех же $f$ и $g$, так и конус $L_{2}^{+}=\left\{f \in L_{2}: f \geqslant 0\right\}$.

Рассмотрим оператор (точнее, линейный ограниченный оператор) $T$, действующий в $L_{2}$. Если конус $L_{2}^{+}$инвариантен относительно $T$ (иными словами, если $T L_{2}^{+} \subset L_{2}^{+}$), условимся говорить, что $f \in L_{2}^{+}$ $T$-мажорирует $g \in L_{2}^{+}$и писать $f \geqslant_{T} g$, коль скоро при некотором выборе $\gamma>0$ и натурального $n$

$$
f+T f+\cdots+T^{n} f \geqslant \gamma g .
$$

При этом запись $f \sim_{T} g$ будет означать, что одновременно $f \geqslant_{T} g$ и $g \geqslant_{T} f$.

Лемма 1. Пусть оператор $T: L_{2} \rightarrow L_{2}$ оставляет конус $L_{2}^{+}$инвариантньи. Тогда отношение $\geqslant_{T}$ транзитивно в $L_{2}^{+}$. Eсли же этот оператор самосопряжен, то для $f, g \in L_{2}^{+}$

$$
\rho(f \vee g)=\rho(f+g)=\max [\rho(f), \rho(g)],
$$

а соотночение $f \geqslant_{T} g$ влечет неравенство $\rho(f) \geqslant \rho(g)$.

Теорема 1. Пусть самосопряженный оператор $T: L_{2} \rightarrow L_{2}$ оставляет конус $L_{2}^{+}$инвариантным, и пусть $f \sim_{Q} g$ для некоторых $f, g \in L_{2}^{+}$, где $Q=T^{2}$, причем $\|T f\| \neq 0$. Если существуют челое $j \geqslant 0$ $u u \in L_{2}^{+}$maxue, umo $g \sim_{Q} a, g \geqslant u u Q^{j} f \geqslant u$, mo

$$
\lim _{n \rightarrow \infty} \frac{\left(Q^{n+1} f, g\right)}{\left(Q^{n} f, g\right)}=\rho^{2}
$$

$c \rho=\rho_{T}(f)$.

По поводу этой теоремы отметим, что ее прообразом служит соответствующий результат работы [13] (чтение которой затруднено из-за обилия искажений). Появление здесь оператора $Q=T^{2}$ связано, в частности, с возможностью эффектов типа периодичности (см. п. 3, следствие 3). По этой же причине без дополнительных предположений из теоремы 1 нельзя извлечь никаких сведений о левой части (1.6) с оператором $Q$, замененным на $T$. 
В оставшейся части пункта семейство рассматриваемых операторов в $L_{2}$ будет несколько сужено, а $\sigma$-алгебра $\mathscr{B}$ будет предполагаться счетнопорожденной. На измеримом пространстве $(E, \mathscr{B})$ мы фиксируем ядро $p(x, d y) \geqslant 0, \sigma$-конечное в смысле [2] вместе со своими итерациями $p_{n}(x, d y), n \geqslant 1$. Для $\mathscr{B}$-измеримых функций $f$ положим $P f(x)=\int f_{+}(y) p(x, d y)+\int f_{-}(y) p(x, d y), x \in E$, коль скоро хотя бы один из выписанных интегралов конечен, где $f_{ \pm}=\frac{1}{2}(|f| \pm f)$. Положим также $\mu P(A)=\int p(x, A) \mu(d x), A \in \mathscr{B}$, для мер $\mu$, заданных на $\mathscr{B}$, и приравняем $\mu(f)$ к интегралу $\int f d \mu$, если он определен.

Будем называть оператор $T: L_{2} \rightarrow L_{2}$ порожденным ядром $p(x, d y)$ или оператором $P$, если для любых $f_{1}$ и $f_{2}$ из одного и того же класса эквивалентности $\tilde{f} \in L_{2}$ функции $P f_{1}$ и $P f_{2}$ входят в класс $T \tilde{f}$. Простое условие, при котором $P$ порождает $T$, см. в лемме 3 из п. 3 . Оператор $T: L_{2} \rightarrow L_{2}$, порожденный заданным ядром, самосопряжен, если это ядро симметрично (точнее, $\xi$-симметрично) в том смысле, что $(P f, g)=(f, P g)$ для всех $\mathscr{B}$-измеримых $f, g \geqslant 0$ (с этого момента символ $(f, g)$, где $f$ и $g$ - вешественные функции на $E$, обозначает также интеграл $\int f g d \xi$, если последний определен).

Ядро $p(x, d y)$, по определению, $\nu$-неприводимо, где $\nu$ - некоторая нетривиальная $\sigma$-конечная мера на $\mathscr{B}$, если $\sum_{n \geqslant 1} p_{n}(x, A)>0$ всюду в $E$ всякий раз, когда $\nu(A)>0[2],[4],[6]$. Симметричное $\nu$-неприводимое ядро является и $\xi$-неприводимым (см. лемму 5), и далее, если это не ведет к недоразумению, используется сокращенный термин «неприводимое ядро».

Вплоть до теоремы 2 ядро $p(x, d y)$ будем предполагать симметричным, неприводимым и порождающим оператор $T: L_{2} \rightarrow L_{2}$. Пусть $\xi_{0}-$ произвольная конечная мера на $\mathscr{B}$, эквивалентная $\xi$. Взяв за основу соответствующее определение из [2], назовем $\mathscr{B}$-измеримую функцию $f \geqslant 0$ минорантной, если $\xi(f)>0$ и $p_{m}(x, A) \geqslant \beta f(x) \xi_{0}(A)$ для $x \in E$ и $A \in \mathscr{B}$ при некоторых $\beta>0$ и натуральном $m$. Если функция-индикатор какого-либо $A \in \mathscr{B}$ минорантна, то и $A$ именуется минорантным, и мы часто будем пользоваться тем, что $A \in \mathscr{B}$ содержит минорантное подмножество, если $\xi(A)>0$ [2], [4].

Как известно [2], при $\xi$-почти всех $x$ степенной ряд

$$
\sum_{n \geqslant 0} t^{n} P^{n} f(x)=G_{t} f(x), \quad t \geqslant 0,
$$

имеет один и тот же конечный радиус сходимости $R$, не зависящий от выбора минорантной функции $f$ и называемый параметром сходимости данного ядра. Мы будем также свободно пользоваться такими понятиями, как $R$-возвратное ядро, где $R$ имеет только что указанный смысл, или период ядра [2]. Ядро $p(x, d y)$ назовем харрисовским, если оно задает 
вероятности перехода некоторой возвратной по Харрису цепи Маркова В $(E, \mathscr{B})$.

При описании связи между $R$ и величинами, определяемыми посредством (1.1), а также и для других целей будем $\mathscr{B}$-измеримые функции с छ-суммируемыми квадратами отождествлять с соответствующими элементами из $L_{2}$. Это позволяет, в частности, величину $\rho(f)$ для функции $f \geqslant 0$ указанного вида определять прежним образом (см. (1.1)), функцию $P f$ отождествлять с $T f$, записи типа $f \geqslant_{P} g$ или $f \sim_{P} g$ истолковывать, как прежде, и т.п.

Предложение 1. Пусть симметричное кеприводимое апериодическое ядро $p(x, d y)$ имеет параметр сходимости $R>0, u$ пусть оно порождает оператор $T: L_{2} \rightarrow L_{2}$. Tогда $f \sim_{P^{2}} g$ для любых минорантных функций $f, g \in L_{2}$. При этом $\rho(f)=R^{-1}$ для $f$ указанного вuдa.

Из нашей теоремы 1 и теоремы 7.4 книги [2] выводится

Теорема 2. Пусть симметричное апериодическое $я д р о ~ p(x, d y)$ является харрисовским. Пусть функции $f, g, u, v$ минорантны $и$ б-суммируемы, причем $\xi(u)>0 u \xi(v)>0$. Тогда при любом челом $m$

$$
\lim _{n \rightarrow \infty} \frac{\left(P^{n} f, g\right)}{\left(P^{n+m} u, v\right)}=\frac{\xi(f) \xi(g)}{\xi(u) \xi(v)} .
$$

Эту теорему можно обобщить следующим образом.

Следствие 1. Пусть функиия $h>0$ является $R$-инвариантной (m.е. $R P h=h$ ) для симметричного апериодического $R$-возвратного ядра $p(x, d y)$. Пусть функции $f, g, u, v$ минорантны $u$ hร-суммируемы, причем $\xi(u)>0 u \xi(v)>0$, где мера $h \xi$ на $\mathscr{B}$ определяется равенстьом $h \xi(d y)=h(y) \xi(d y)$. Тогда при челых $m$

$$
\lim _{n \rightarrow \infty} \frac{\left(P^{n} f, g\right)}{\left(P^{n+m} u, v\right)}=R^{m} \frac{\xi(f h) \xi(g h)}{\xi(u h) \xi(v h)} .
$$

Что касается существования упомянутой здесь функции $h$, то оно гарантируется $R$-возвратностью рассматриваемого ядра [2]. Заметим также, что симметричное неразложимое ядро либо апериодично, либо ммеет период $d=2$ (см. следствие 3 ). Поэтому не составляет труда лереформулировать теорему 2 и следствие 1 так, чтобы они годились и в периодическом случае (ср. [12]).

Особый интерес вызывает случай локально компактного пространства $E$ (эргодическая теория для феллеровских стохастических ядер на компактных пространствах представлена результатами Бебутова, Иосиды и Крылова-Боголюбова - см., например, [15] - и мы не будем к ней обращаться). До конца пункта мы постулируем следующее условие: 
(I) $E$ является локально компактным пространством со счетной базой, $\mathscr{B}$ - его борелевской $\sigma$-алгеброй, $\xi$ - мерой Радона (т.е. мерой на $\mathscr{B}$ с конечными значениями на компактах), плотной в $E$.

Требование плотности $\xi$ (согласно нему $\xi(U)>0$ для непустых открытых $U \subset E$ ) малосушественно: можно выделить такое замкнутое $E^{\prime} \subset E$ с $\xi\left(E \backslash E^{\prime}\right)=0$, что сужение меры $\xi$ на $E^{\prime}$ плотно в $E^{\prime}$, и вместо $E$ рассмотреть $E^{\prime}$, заменив $\xi$ и $p(x, d y)$ соответствующими сужениями. Символом $C_{0}^{+}$обозначается совокупность всех непрерывных в $E$ функций $f \geqslant 0$ с компактньми носителями, исключая $f \equiv 0$. Ядро $p(x, d y)$ и отвечающий ему оператор $P$ назовем квазифеллеровскими, если непрерывны все функции вида $P^{n} f$, где $f \in C_{0}^{+}, n \geqslant 1$. Именно так обстоит дело, если функции вида $P f, f \in C_{0}^{+}$, и функции $p_{n}(\cdot, E), n \geqslant 1$, непрерывны [13].

Как и при изучении цепей Маркова на топологических пространствах (см., например, [7]), введем условие «топологической связности»:

(II) для любых $x \in E$ и $f \in C_{0}^{+}$реализуется неравенство $P^{n} f(x)>0$ при каком-либо $n \geqslant 1$ (зависящем, вообще говоря, от $f$ и $x$ ).

Впоследствии будем говорить о выполнении условия (IIa) (соответственно (IIб)), если указанное в (II) число $n$ можно считать четным (нечетным). Во многих случаях (IIa) $\Longrightarrow$ (IIб) (см. п. 5), и условие (IIa) позволит в теореме 3 обойтись без требований типа апериодичности или неприводимости.

Ради краткости квазифеллеровское симметричное ядро $p(x, d y)$, подчиненное условию (IIa) и порождающее оператор $T: L_{2} \rightarrow L_{2}$, назовем допустимым. Поскольку для такого ядра величина $\rho(f)=\rho_{T}(f)$ (см. (1.1)) не зависит от выбора $f \in C_{0}^{+}$(см. п. 5), ему можно сопоставить параметр

$$
r=[\rho(f)]^{-1}=\lim _{n \rightarrow \infty}\left[\left\|P^{n} f\right\|^{-1 / n}\right], \quad f \in C_{0}^{+},
$$

не зависящий от конкретного выбора $f$, причем $0<r<\infty$ в соответствии с (1.3) (согласно (IIa) $\|T f\|>0$ для $f \in C_{0}^{+}$).

Предложение 2. Если $R$ - параметр сходимости неприводимого допустимого ядра $p(x, d y)$, то $R \geqslant r$. При этом $R=r$, коль скоро хотя бы одна из функций $f \in C_{0}^{+}$является минорантной.

Допустимое ядро $p(x, d y)$ и отвечаюший ему оператор $P$ назовем топологически лиувиллевыми, если любые две нетривиальные меры Радона, $r$-инвариантные для $P$ (т.е. являющиеся решениями уравнения $r \mu P=\mu)$, могут отличаться друг от друга лишь постоянным множителем. При $r=1$ свойство топологической лиувиллевости исследовалось, например, в [7]. 
Введем еще одно условие:

(III) либо $E$ компактно, либо для каждой функции $f \in C_{0}^{+}$найдутся $\left.\varphi \in C_{0}^{+}, t \in\right] 0, r[$ и натуральное $l$, при которых

$$
\lim _{x \rightarrow \infty} \frac{P^{2 l} f(x)}{H_{t} \varphi(x)}=0
$$

где положено $H_{t} \varphi=\sum_{n \geqslant 1} t^{2 n} P^{2 n} \varphi$ (здесь имеется в виду обычная одноточечная компактификация пространства $E$ ).

Для допустимого ядра $p(x, d y)$ условие (III) заведомо выполнено, если соответствующий оператор $P$ отображает $C_{0}^{+}$в себя. ПІрименительно к допустимому субстохастическому ядру соотношение (1.10) можно истолковать, как требование, чтобы из дополнения достаточно большого компакта в $E$ цепь Маркова с переходными вероятностями $p_{2}(x, d y)$ попадала на $l$-м шаге в компактный носитель функции $f$ с несравненно меньшей вероятностью, чем в другой (возможно, больший) компакт за, быть может, большее число шагов.

Теорема 3. Пусть выполнено условие (I). Пусть ядро $p(x, d y)$ допустимо и удовлетворяет условию (III), а ядро $p_{2}(x, d y)$ является топологически лиувиллевым. Тогда, каковы бы ни были функции $f, g, u, v \in C_{0}^{+}$и натуральное $m$,

$$
\frac{\left(P^{n} f, g\right)}{\left(P^{n+m} f, g\right)} \longrightarrow r^{m} \frac{\eta(f) \eta(g)}{\eta(u) \eta(v)} \quad \text { npu } n \rightarrow \infty
$$

где $r$ - то же, что и в (1.9), а $\eta$ - произвольная нетривиальная $r^{2}$-инвариантная мера Радона для ядра $p_{2}(x, d y)$.

Теорема 3, в частности, применима к ситуации, когда в $(E, \mathscr{B})$ задана (возможно, обрываюшаяся) цепь Маркова с переходными вероятностями $p(x, d y)$, удовлетворяющими требованиям теоремы. При этом лемма 2 п. 2 снимает вопрос о сушествовании соответствующего оператора $T$. Если же указанная цепь не обрывается и $r=1$, то мера $\xi$ инвариантна и для ядра $p(x, d y)$, и для ядра $p_{2}(x, d y)$ (см. лемму 3 в п. 3), так что в этом случае в (1.11) можно считать $\eta=\xi$ и опустить множитель $r^{m}$.

С помощью теоремы 3 легко получить упоминавшуюся предельную теорему для отношений, порожденных простым $d$-мерным случайным блужданием при $d \geqslant 1[14]$. С этой целью теорему 3 надо применить не к соответствующему ядру $p(x, d y)$, а к сужению $p_{2}(x, d y)$ на один из двух циклических классов. Отметим, что случай $d \geqslant 3$ не может быть рассмотрен ни с помощью уже обсуждавшейся теоремы Ори, ни с помошью нашей теоремы 2.

В связи с теоремой 3 возникает естественный вопрос: когда лиувиллевость ядра $p(x, d y)$ влечет аналогичное свойство для $p_{2}(x, d y) ?$ Частичный ответ можно найти в п. 6 . 
2. K теореме 1. В этом пункте фиксированы измеримое пространство $(E, \mathscr{B}), \sigma$-конечная мера $\xi$ на $\mathscr{B}$ и линейный ограниченный оператор $T: L_{2} \rightarrow L_{2}$, оставляющий инвариантным конус $L_{2}^{+}$. Наша цель состоит в доказательстве теоремы 1 , для чего требуется определенная подготовка.

Доказательст тво лем мы 1 . Пусть $f_{i} \in L_{2}^{+}, 1 \leqslant i \leqslant 3$, и $f_{i} \geqslant f_{i+1}$ при $i=1,2$, т.е. $f_{i}+T f_{i}+\cdots+T^{k} f_{i} \geqslant \gamma f_{i+1}$ при $i=1,2$ и соответствующих $\gamma>0$ и $k \geqslant 1$ (cp. (1.4)). Тогда

$$
\gamma^{2} f_{3} \leqslant \sum_{0 \leqslant m \leqslant k} T^{m}\left(\sum_{0 \leqslant j \leqslant k} T^{j} f_{1}\right) \leqslant \alpha \sum_{0 \leqslant m \leqslant 2 k} T^{m} f_{1},
$$

где $\alpha>0$, откуда и следует транзитивность отношения $\geqslant_{T}$.

Далее оператор $T$ считается самосопряженным. Пусть $f, g \in L_{2}^{+}$и $\rho(f) \geqslant \rho(g)$. Так как $\rho(f) \leqslant \rho(f \vee g) \leqslant \rho(f+g)$, для доказательства (1.5) достаточно установить лишь второе из равенств в (1.5). Неравенства

$$
\rho(f) \leqslant \rho(f+g) \leqslant \limsup _{n \rightarrow \infty}\left\{\left(\left\|T^{n} f\right\|+\left\|T^{n} g\right\|\right)^{1 / n}\right\}
$$

очевидны при $\|T f\|=0$ и вытекают из (1.1) при $\|T f\| \neq 0$. Если же $s>\rho(f)$ и $n$ достаточно велико, то $\left\|T^{n} f\right\|+\left\|T^{n} g\right\|<2 s^{n}$, так что $\rho(f+$ $g) \leqslant s$ и второе из равенств (1.5) доказано.

Пусть $f \geqslant_{T} g$, а $h$ равно левой части (1.4). Соотношения (1.5) и (1.2) показывают, что $\rho(f)=\rho(h) \geqslant \rho(g)$, и лемма доказана.

Лемма 2. Пусть оператор $T$ самосопряжен $u f, g \in L_{2}^{+}$, причем $g \geqslant_{T^{2}} f$. Тогда при подходящем $C_{1}>0$

$$
\left\|T^{n} f\right\| \leqslant C_{1}\left\|T^{n} g\right\|, \quad n \geqslant 0 .
$$

Eсли $\|T g\| \neq 0 u T^{2 j} f \geqslant a g$ при некоторых $a>0 u j \geqslant 0$, mо прu подходящих $C_{2}, C_{3}>0$

$$
C_{2} \leqslant\left(T^{2 n} f, g\right)\left\|T^{n} g\right\|^{-2} \leqslant C_{3}, \quad n \geqslant j .
$$

Если же $T^{2 l+1} f \geqslant b g$ при каких-либо $b>0$ u $l \geqslant 0$, то оченки типа (2.3) верны и для $\left(T^{2 n+1} f, g\right)\left\|T^{n} g\right\|^{-2}$.

Д ок а зат ел ьс т в о. Если $\|T g\|=0$, то, как легко заметить, и $\|T f\|=0$, и оценка (2.2) очевидна. Поэтому будем считать, что $\|T g\| \neq 0$. По аналогии с (1.4) $g+T^{2} g+\cdots+T^{2 k} g \geqslant \gamma f$ при соответствующих $\gamma>0$ и $k \geqslant 1$, и, таким образом,

$$
\begin{aligned}
\left(T^{2 n} f, f\right) & \leqslant \gamma^{-2} \sum_{0 \leqslant m, i \leqslant k}\left(T^{2 n+2 m} g, T^{2 i} g\right) \\
& =\gamma^{-2} \sum_{0 \leqslant m, i \geqslant k}\left\|T^{n+m+i} g\right\|^{2} \leqslant C_{4} \sum_{0 \leqslant m \leqslant 2 k}\left\|T^{n+m} g\right\|^{2} \leqslant C_{5}\left\|T^{n} g\right\|^{2}
\end{aligned}
$$

с некоторыми $C_{4}, C_{5}>0$ (см. (1.3)). Отсюда вытекает (2.2). 
Если же $T^{2 j} f \geqslant a g$ и $n \geqslant j$, то $\left\|T^{n+j} f\right\|^{2}$ совпадает с

$$
\left(T^{2 n} f, T^{2 j} f\right) \geqslant a\left(T^{2 n} f, g\right) \geqslant a^{2}\left(T^{2 n-2 j} g, g\right)=a^{2}\left\|T^{n-j} g\right\|^{2} .
$$

Отношения величин $\left\|T^{n+j} f\right\|$ и $\left\|T^{n-j} f\right\|$ к $\left\|T^{n} g\right\|$ в согласии с (1.3) и (2.2) заключены между двумя положительными константами, и ввиду выписанной цепочки соотношений то же можно сказать и о величине $\left(T^{2 n} f, g\right)\left\|T^{n} g\right\|^{-2}$. Тем самым (2.3) доказано.

Если же существуют $l$ и $b$, указанные в лемме, то при $n \geqslant l$

$$
\grave{b}\left(T^{2 n-2 l} g, g\right) \leqslant\left(T^{2 n+1} f, g\right) \leqslant\left\|T^{n} f\right\| \cdot\left\|T^{n+1} g\right\| \leqslant C_{1}\|T\| \cdot\left\|T^{n} g\right\|^{2},
$$

и по аналогии с предыдущим получаем последнее утверждение леммы.

Следствие 2. Пусть оператор $T$ самосопряжен $u f, g \in L_{2}^{+}, n p u-$ чем $f \sim_{T^{2}} g$ и $\|T f\| \neq 0$. Тогда последовательность $\left\{\left\|T^{n} f\right\| \cdot\left\|T^{n} g\right\|^{-1}\right.$; $n \geqslant 0\}$, ограничена. Если же $T^{2 j} f>$ ag при некоторых $a>0 u j \geqslant 0$, то и последовательность $\left\{\left\|T^{n} f\right\|^{2}\left(T^{2 n} f, g\right)^{-1} ; n \geqslant 1\right\}$ ограничена.

Доказательство те оремы 1. Ограничимся случаем $\rho=1$ (иначе следовало бы $T$ заменить на $\rho^{-1} T$ ). Пусть $h \equiv f+Q f+$ $\cdots+Q^{k} f \geqslant \gamma g$ (cр. (1.4)), $\gamma>0, w=\gamma g, f_{1}=h+w$ и $f_{2}=h-w$. Ясно, что $h \sim_{Q} f \sim_{Q} w \sim_{Q} u$ и $\rho\left(f_{1}\right)=\rho(h)=\rho(w)=\rho=1$ (см. лемму 1 ). Увеличив при необходимости $k$, добьемся, чтобы и $\rho\left(f_{2}\right)=\rho(h)=1$ (см. (1.5)).

Последующие рассуждения проведем в три этапа.

$1^{\circ}$. Начнем с вывода оценки

$$
\alpha_{n} \equiv \frac{\left(Q^{n} h, h\right)}{\left(Q^{n} f, w\right)} \leqslant C, \quad n \geqslant j
$$

при некотором $C>0$. Рассмотрим отношения:

$$
I_{1, n}=\frac{\left(Q^{n} h, h\right)}{\left(Q^{n} f, f\right)}, \quad I_{2, n}=\frac{\left(Q^{n} f, f\right)}{\left(Q^{n} f, u\right)}, \quad I_{3, n}=\frac{\left(Q^{n} f, u\right)}{\left(Q^{n} f, w\right)}, \quad n \geqslant j .
$$

Величины вида $I_{1, n}$ или $I_{2, n}$ по следствию 2 не превышают некоторой постоянной. То же относится и к $I_{3, n}$, поскольку $w=\gamma g \geqslant \gamma u$. Отсюда и из представления $\alpha_{n}=I_{1, n} I_{2, n} I_{3, n}$ получаем (2.4).

$2^{\circ}$. Теперь докажем равенство

$$
\lim _{n \rightarrow \infty} \frac{\left(Q^{n+1} h, w\right)}{\left(Q^{n} h, w\right)}=1
$$

Используя определение $f_{1}$ и $f_{2}$ и учитывая самосопряженность $Q$, находим

$$
\left(Q^{n} f_{1}, f_{1}\right)-\left(Q^{n} f_{2}, f_{2}\right)=4\left(Q^{n} h, w\right)
$$


Пусть $0<\varepsilon<1$. Из равенств $\rho\left(f_{1}\right)=\rho\left(f_{2}\right)=1$ и из (1.1) следует, что

$$
1-\varepsilon<\left(Q^{n+1} f_{i}, f_{i}\right)\left(Q^{n} f_{i}, f_{i}\right)^{-1}<1+\varepsilon, \quad i=1,2,
$$

при $n$, превышающем некоторое натуральное $n_{1} \geqslant j$ (ведь $\left(Q^{n} f_{i}, f_{i}\right)=$ $\left.\left\|T^{n} f_{i}\right\|^{2}\right)$. Поэтому, дважды применив (2.6), при $n>n_{1}$ получаем

$$
\begin{aligned}
4\left(Q^{n+1} h, w\right) & \leqslant(1+\varepsilon)\left(Q^{n} f_{1}, f_{1}\right)-(1-\varepsilon)\left(Q^{n} f_{2}, f_{2}\right) \\
& =(1+\varepsilon)\left[\left(Q^{n} f_{1}, f_{1}\right)-\left(Q^{n} f_{2}, f_{2}\right)\right]+2 \varepsilon\left(Q^{n} f_{2}, f_{2}\right) \\
& \leqslant 4(1+\varepsilon)\left(Q^{n} h, w\right)+2 \varepsilon\left(Q^{n} h, h\right)
\end{aligned}
$$

и аналогично

$$
\begin{aligned}
4\left(Q^{n+1} h, w\right) & \geqslant(1-\varepsilon)\left(Q^{n} f_{1}, f_{1}\right)-(1+\varepsilon)\left(Q^{n} f_{2}, f_{2}\right) \\
& \geqslant 4(1-\varepsilon)\left(Q^{n} h, w\right)-2 \varepsilon\left(Q^{n} h, h\right) .
\end{aligned}
$$

В силу этих оценок отношение $\left(Q^{n+1} h, w\right) /\left(Q^{n} h, w\right)$ заключено между $1-\varepsilon-\varepsilon a_{n}$ и $1+\varepsilon+\varepsilon a_{n}$, где $a_{n} \equiv\left(Q^{n} h, h\right)\left(Q^{n} h, w\right)^{-1} \leqslant \alpha_{n} \leqslant C$ (см. (2.4)) и $n>n_{1}$. Теперь (2.5) становится очевидным.

$3^{\circ}$. Заметим, что

$$
\lim _{n \rightarrow \infty} a_{n, k}=1
$$

где $a_{n, k}=\left(Q^{n+k+1} f, w\right)\left(Q^{n} f, w\right)^{-1}$ (до конца доказательства считается, что $n>j$ ). Действительно, вспомнив определение $h$ и применив (2.5), находим

$$
\frac{a_{n, k}-1}{b_{n}}=\frac{\left(Q^{n+k+1} f, w\right)-\left(Q^{n} f, w\right)}{\left(Q^{n} h, w\right)}=\frac{\left(Q^{n+1} h, w\right)-\left(Q^{n} h, w\right)}{\left(Q^{n} h, w\right)} \rightarrow 0
$$

где $b_{n} \equiv\left(Q^{n} h, w\right)\left(Q^{n} f, w\right)^{-1} \leqslant \alpha_{n} \leqslant C$ (см. (2.4)), откуда следует (2.8).

Настал момент заметить, что при выборе $\gamma>0$ и $k$ мы требовали лишь осушествления соотношений $f+Q f+\cdots+Q^{k} f \geqslant \gamma g$ и $\rho\left(f_{2}\right)=1$. Ввиду этого предыдущие рассуждения остаются в силе и при замене пары $\gamma$ и $k$ парой $\gamma$ и $k+1$. Иными словами, (2.8) можно использовать и при $k$, замененном на $k+1$, и в итоге

$$
1=\lim _{n \rightarrow \infty} \frac{a_{n, k}}{a_{n, k+1}}=\lim _{n \rightarrow \infty} \frac{\left(Q^{n+k+1} f, w\right)}{\left(Q^{n+k+2} f, w\right)} .
$$

Таким образом, соотношение (1.6) доказано.

3. Некоторые свойства неотрицательных ядер. На протяжении этого пункта рассматривается измеримое пространство $(E, \mathscr{B})$ со счетнопорожденной $\sigma$-алгеброй $\mathscr{B}$, на которой определена $\sigma$-конечная мера $\xi$. На этом пространстве задается ядро $p(x, d y) \geqslant 0$, являющееся 
$\sigma$-конечным вместе со своими итерациями, и с ним ассоциируется оператор $P$ (см. п. 1 ).

В параграфе представлен довольно разнородный материал, необходимый для дальнейшего. Наибольший интерес здесь представляют следствие 3 и доказательство предложения 1. Начинаем же мы с утверждения, различные варианты которого встречались в литературе (см., например, [13]).

Лемма 3. Пусть ядро $p(x, d y)$ ограничено: $p(\cdot, E) \leqslant C$ при некотором $C \geqslant 0$. Если мера $\xi \alpha$-субинвариантна (т.е. $\alpha \xi P \leqslant \xi)$ при некотором $\alpha>0$, то это ядро порождает линейный ограниченный оператор $T: L_{2} \rightarrow L_{2}$ с нормой $\|T\| \leqslant(C / \alpha)^{1 / 2}$.

Д ок а з а т л в с т о. По неравенству Коши-Буняковского

$$
\int(P f)^{2} d \xi \leqslant \int[P 1]\left[P\left(f^{2}\right)\right] d \xi \leqslant C \int f^{2} d(\xi P) \leqslant C \alpha^{-1} \int f^{2} d \xi
$$

для любых неотрицательных $\mathscr{B}$-измеримых функций, что и требовалось доказать.

Напомним, что $\xi$-симметричные ядра мы называем симметричными, а запись «п.в.» означает « $\xi$-почти вскоду.

Лемма 4. Пусть ядро $p(x, d y)$ субстохастично и симметрично. Тогда мера $\xi$ субинвариантна относительно него, причем она инвариантна тогда и только тогда, когда $p(\cdot, E)=1$ n.в.

Д ок а з а т ел ь с т в о. Субинвариантность вытекает из соотношений $\int P f d \xi=\int f \cdot P 1 d \xi \leqslant \int f d \xi$, в которых функция $f \geqslant 0$ предполагается $\mathscr{B}$-измеримой. Присутствующее здесь равенство влечет и второе утверждение леммы.

При $A \in \mathscr{B}$ и $t>0$ положим

$$
G(\cdot, A)=\sum_{n \geqslant 1} p_{n}(\cdot, A) \quad \text { и } \quad A^{+}=A \cup\{x \in E: G(x, A)>0\} .
$$

Лемма 5. Пусть ядро $p(x, d y)$ симметрично и $\nu$-неприводимо, где $\nu$ - некоторая $\sigma$-конечная мера на $\mathscr{B}$. Тогда данное ядро $\xi$-неприводимо, причем $\xi\left(A^{+}\right)=0$, если $A \in \mathscr{B}$ и $\xi(A)=0$.

Д ок а з т е л с т в о. В силу симметричности рассматриваемого ядра

$$
\int_{B} G(x, A) d \xi=\int_{A} G(x, B) d \xi
$$

для $A, B \in \mathscr{B}$. При $B=E$ и $\xi(A)=0$ из (3.1) усматриваем, что $G(\cdot, A)=0$ п.в., и, следовательно, $\nu(A)=0$ в соответствии с условием $\nu$-неприводимости. Иными словами, мера $\nu$ абсолютно непрерывна относительно $\xi$ и существует плотность $\varphi=d \nu / d \xi$, которую из-за $\sigma$-конечности $\xi$ допустимо считать конечной всюду. 
Не ограничивая общности, предположим, что $\nu\left(A^{+}\right)=0$ всякий раз, когда $\nu(A)=0[2],[6]$. Так как $\nu(B)=0$ для $B=\{x \in E: \varphi(x)=0\}$, то и $\nu\left(B^{+}\right)=0$. Для $x \in A \equiv E \backslash B^{+}$имеем $G\left(x, B^{+}\right)=0$, тогда как $G(\cdot, A)>0$ всюду в $E$ ввиду очевидного неравенства $\nu(A)>0$. В этой ситуации (3.1) с $B^{+}$вместо $B$ показывает, что $\xi\left(B^{+}\right)=0$, а потому $G(\cdot, B)=0$ п.в. Очередное применение (3.1) с $A=E$ ведет к равенству $G(\cdot, E)=0$ п.в. на $B$, вследствие которого $\xi(B)=0$ (ведь условие $\nu$-неприводимости исключает существование $x \in E$ с $G(x, E)=0)$. Таким образом, $\varphi=d \nu / d \xi>0$ п.в. и мера $\xi$ оказывается аббсолютно непрерывной относительно $\nu$, что и завершает доказательство.

Лемма 5 оправдывает использование (см. п. 1) термина «неприводимое ядро» применительно к $\xi$-неприводимым или $\nu$-неприводимым симметричным ядрам. Более того, в случае симметричного неприводимого ядра из первого абзаца доказательства леммы вытекает, что $\xi$ является максимальной мерой, определяющей неприводимость [2].

Следствие 3. Если ядро $p(x, d y)$ симметрично и неприводимо, то оно имеет период $d=1$ или 2.

Действительно, при $d>2$ нашлось бы множество $A \in \mathscr{B} \mathrm{c} \xi(A)>0$, во всех точках $x$ которого $P^{2} 1_{A}(x)=0$, где $1_{A}-$ функция-индикатор этого множества. Но тогда из равенств $\int\left(P^{n} 1_{A}\right)^{2} d \xi=\left(P 1_{A}, P^{2 n-1} 1_{A}\right)=$ $\left(1_{A}, P^{2 n} 1_{A}\right)$ при $n=1$ мы сначала вывели бы равенство $P 1_{A}=0$ п.в., а затем при $n \geqslant 2$ и равенство $P^{n} 1_{A}=0$ п.в., что несовместимо с неприводимостью ядра.

Док азат ельст во предложения 1. Если функции $f, g \in L_{2}$ минорантны, то

$$
P^{n+m} f \geqslant \beta \gamma_{n} g
$$

при некоторых $\beta>0$ и $m \geqslant 1$ и всех $n \geqslant 0$, где $\gamma_{n}=\int P^{n} f d \xi_{0}$ (см. доказательство предложения 2.7 из [2]; здесь $\xi_{0}$ - мера, вводившаяся в п. 1 при определении минорантных функций). Ввиду апериодичности данного ядра $\gamma_{n}>0$ при всех достаточно больших $n$. Стало быть, $f \geqslant_{P^{2}} g$, а так как $f$ и $g$ можно поменять ролями, то $f \sim_{P^{2}} g$ и $\rho(f)=\rho(g)$ (см. лемму 1).

Докажем, что для любой минорантной функции $f$

$$
\liminf _{n \rightarrow \infty}\left[P^{n} f\right]^{1 / n} \geqslant R^{-1} \quad \text { (п.в.). }
$$

В самом деле, если она является индикатором минорантного множества, то (3.3) вытекает из результатов работы [9] (в ней речь идет о субстохастическом случае, однако те же соображения применимы в общей ситуации (cp. $[6, \S 6]))$. Для произвольной минорантной функции $f$ можно подобрать минорантное множество $A$, на котором $f \geqslant a$ при некотором $a>0$. Отсюда вновь следует (3.3). 
Теперь для минорантной функции $f \in L_{2}$ установим неравенство

$$
\rho(f) \geqslant R^{-1} \text {. }
$$

Рассмотрим множества $A_{n}=\cap_{m \geqslant n}\left\{x \in E: P^{m} f(x) \geqslant \alpha^{m}\right\}, n \geqslant 1$, при фиксированном $\alpha \in] 0, R^{-1}$. Поскольку $A_{1} \subset A_{2} \subset \cdots$ и $\xi\left(E \cup_{n \geqslant 1} A_{n}\right)=0$ (см. (3.3)), все значения $\xi\left(A_{n}\right)$, начиная с некоторого номера $n$, превышают некое $b>0$. Пользуясь неприводимостью рассматриваемого ядра, найдем натуральные $i$ и $j$, при которых $\xi(B)>0$, где $B=\{x \in E$ : $\varphi(x)>0\}$, а $\varphi(\cdot)=p_{i}\left(\cdot, A_{j}\right)$. Затем подберем множество $B_{1} \subset B$ с $\xi\left(B_{1}\right)>0$, на котором $\varphi \geqslant c$ при некотором $c>0$. Для $x \in B_{1}$

$$
P^{n+i} f(x) \geqslant \int_{A_{j}} P^{n} f(y) p_{i}(x, d y) \geqslant \alpha^{n} \varphi(x) \geqslant c \alpha^{n}, \quad n \geqslant j .
$$

Следовательно, $\left\|P^{n+i} f\right\|^{2} \geqslant c^{2} \alpha^{2 n} \xi\left(B_{1}\right), n \geqslant j$ и $\rho(f) \geqslant \alpha$ (см. (1.1)). Тем самым, (3.4) доказано, так как $\alpha$ можно взять сколь угодно близким к $R^{-1}$.

На основании сказанного в начале доказательства остается предъявить какую-нибудь минорантную функцию $f \in L_{2}$ с $\rho(f)=R^{-1}$. Для этого вернемся к мере $\xi_{0}$ и положим $\psi=d \xi_{0} / d \xi \geqslant 0$, а в качестве $f$ возьмем минорантную функцию из $L_{2}$, не превышающую $\psi$. Воспользовавшись (3.2) с $g=f$, находим

$$
P^{n+m} f \geqslant \beta f \int P^{n} f d \xi_{0} \geqslant \beta f \int P^{n} f \cdot f d \xi=\beta\left(P^{n} f, f\right) f .
$$

Если допустить, что $\rho_{1} \equiv \rho(f)>R^{-1}$, то при любом $\left.t \in\right] \rho_{1}^{-1}, R[$ степенной ряд

$$
\sum_{n \geqslant 1} t^{2 n}\left\|P^{n} f\right\|^{2}=\sum_{n \geqslant 1} t^{2 n}\left(P^{2 n} f, f\right)
$$

должен расходиться. В силу последней цепочки соотношений должен разойтись и ряд $\sum_{n \geqslant 1} t^{2 n} p^{2 n+m} f(x)$ при тех $x$, в которых $f(x)>0$. Таким образом, $t \geqslant R$, но это исключено для теперешних значений $t$, и сделанное предположение приходится отбросить. Итак, $\rho(f)=R^{-1}$.

4. Доказательство теоремы 2. Пусть $p(x, d y)$ удовлетворяет условиям доказываемой теоремы. Если функции $f, g \in L_{2}$ минорантны, то $f \sim_{P^{2}} g$ (см. доказательство предложения 1). Умножив функциюиндикатор $u_{1}$ какого-либо минорантного множества, на котором $g \geqslant c$ при каком-нибудь $c>0$, на подходящий множитель, получим минорантную функцию $u_{2} \leqslant g$. При этом в соответствии с (3.2) одновременно сможем добиться и выполнения неравенства $P^{2 j} f \geqslant u_{2}$ при некотором $j \geqslant 1$. На основании теоремы 1 , положив $Q=P^{2}$, получаем

$$
\lim _{n \rightarrow \infty} \frac{\left(Q^{n+1} f, g\right)}{\left(Q^{n} f, g\right)}=1
$$

Как нетрудно заметить, цепь Маркова с переходным оператором $Q$ является неприводимой. Если бы ее параметр сходимости превысил 1 
или равнялся 1 , но она не была бы 1-возвратной, то потенциалы вида $H w=\sum_{n \geqslant 1} Q^{n} w$ были бы ограничены для достаточно большого семейства функций $w$ (см. [2, следствие 3.1]). Тогда и потенциалы вида $G w=\sum_{n \geqslant 1} P^{n} w=H w+P H w$ обладали бы тем же свойством, а это исключается возвратностью основной цепи. Следовательно, цепь Маркова, отвечающая $Q$, является 1-возвратной, и в соответствии с утверждениями 3.7 и 3.13 из [2] найдется множество $E_{1} \in \mathscr{B}$, сужение (в другой терминологии - часть) на котором этой цепи возвратно по Харрису. Так как $\xi\left(E \backslash E_{1}\right)=0$, можно безболезненно допустить, что $E=E_{1}$, т.е. что сама эта цепь возвратна по Харрису (иначе в дальнейших рассуждениях потребовались бы микроскопические изменения).

Пусть имеются $\xi$-суммируемые минорантные для основного ядра функции $f, g, u, v$. Будучи ограниченными, они автоматически входят в $L_{2}$. Ввиду (4.1) $\lim _{n \rightarrow \infty} a_{n+1} a_{n}^{-1}=1$, если $a_{n}=\left(Q^{n} f, \widetilde{g}\right)$, где $\xi(\widetilde{g})=1$ и $\tilde{g}$ отличается от $g$ лишь подходящим множителем. Поэтому к оператору $T_{1}: L_{2} \rightarrow L_{2}$, ассоциированному с $Q$, и к мере $\nu=\widetilde{g} \xi$ применима теорема 7.4 из [2], согласно которой $\left(Q^{n} f, g\right)\left(Q^{n} v, g\right)^{-1} \longrightarrow \xi(f)[\xi(v)]^{-1}$ при $n \rightarrow \infty$ (с помошью (3.2) нетрудно убедиться в том; что функция, минорантная для основной цепи, минорантна и для цепи, отвечающей $Q$ ). Точно так же $\left(Q^{n} g, v\right)\left(Q^{n} u, v\right)^{-1} \longrightarrow \xi(g)[\xi(u)]^{-1}$, и в итоге

$$
\frac{\left(Q^{n} f, g\right)}{\left(Q^{n} u, v\right)} \rightarrow \frac{\xi(f) \xi(g)}{\xi(u) \xi(v)}
$$

при $n \rightarrow \infty$. Вместе с $f$ минорантной является и функция $P f$, и возникает возможность использовать (4.2) с $P f$ вместо $f$, причем $\xi(P f)=\xi(f)$ по лемме 4 . В сочетании с (4.2) это дает (1.8).

Доказательство след с твия 1 (набросок). Траектория цепи Маркова, отвечаюшей $P$, почти наверное не попадает в дополнение множества $E^{\prime}=\{x: h(x)<\infty\}$, если она начинается в этом множестве. При этом $\xi\left(E \backslash E^{\prime}\right)=0$, так что доказательство достаточно провести для сужения цепи на $E^{\prime}$, с учетом чего мы ограничиваемся случаем $E=E^{\prime}$. C помощью «преобразования подобия» [2] введем новое ядро $\widetilde{p}(x, d y)=h^{-1}(x) h(y) p(x, d y), x \in E$, симметричное относительно меры $\eta=h^{2} \xi$. Как показывает непосредственная проверка, всякая функция вида $\varphi / h$ является минорантной для нового ядра, если $\psi$ минорантна для исходного (для нового ядра в роли меры $\xi_{0}$ из прежнего определения минорантной функции можно взять $\tilde{\xi}_{0}=h_{1} \xi_{0}$ с $\left.h_{1}=\min (h, 1)\right)$.

Поскольку новое ядро является 1-возвратным, можно предположить его харрисовским (ср. с предыдущим доказательством). Это позволяет применить вариант (1.8), соответствующий замене $\xi$ на $h, p(x, d y)$ на $\tilde{p}(x, d y)$ и функций $f, g$ и т.д. на $f / h, g / h$ и т.д. В результате и будет доказано утверждение следствия. 
5. Случай квазифеллеровского ядра. Всюду в этом пункте постулируется условие (I) из п. 1 и на измеримом пространстве $(E, \mathscr{B})$ задается допустимое в смысле п. 1 ядро $p(x, d y) \geqslant 0$ (т.е. квазифеллеровское симметричное ядро, подчиненное условию (IIa) и порождающее оператор $T: L_{2} \rightarrow L_{2}$; эти условия, как правило, не оговариваются). Постоянно используется обозначение $Q=P^{2}$.

Основная цель состоит в доказательстве теоремы 3 (см. 1), однако сначала необходимо установить корректность определения параметра $r>0$ посредством (1.9) (см. следствие 4 ниже) и доказать предложение 2.

Сразу подчеркнем, что при наших предположениях условия (II) и (IIб) равносильны. Действительно, в силу (IIa) $\varphi(\cdot) \equiv \sum_{i \geqslant 1} Q^{i} f(\cdot)>0$ в $E$ для $f \in C_{0}^{+}$, и, следовательно, $P \varphi(\cdot)>0$. Таким образом, (ІІа) $\Rightarrow$ (ІІб), и аналогично (ІІб) $\Rightarrow$ (IІб).

Лемма 6. При любых $f, g \in C_{0}^{+} u$ челых $k, m \geqslant 0$ реализуется соотношение $P^{m} f \sim_{Q} P^{k} g$.

Д ок азат е льст в о. Компакты $F_{n}=\left\{x \in F: P^{m} s_{n}(x)=0\right\}$, где $F$ - компактный носитель функции $g$ и $s_{n}=\sum_{1 \leqslant i \leqslant n} Q^{i} f$, с ростом $n \geqslant 1$ не возрастают, а на их пересечении должно выполняться равенство $P^{m}\left[\sum_{i \geqslant 1} Q^{i} f\right]=0$, исключаемое условием (IIa). Следовательно, это пересечение пусто, а с ним, в силу (I), пуст и компакт $\cap_{1 \leqslant i \leqslant j} F_{i}$ при некотором натуральном $j$. Поэтому $P^{m} s_{j} \geqslant a g$, если $a>0$ достаточно мало, т.е. $P^{m} f \geqslant_{Q} g$, и аналогично $P^{k} g \geqslant_{Q} f$, так что $f \sim_{Q} g$ и $P^{k} s_{n} \geqslant g$ при подходяшем $n$. Применение оператора $P^{k}$ к обеим частям последнего неравенства показывает, что $f \geqslant_{Q} P^{k} g$. Таким образом, $f \sim_{Q} P^{k} g$ и точно так же $g \sim_{Q} P^{m} f$, и утверждение леммы становится очевидным.

Из доказанной леммы непосредственно вытекает

Следствие 4. Значения $\rho\left(P^{m} f\right)$, а с ними и значения $r\left(P^{m} f\right)=$ $\left[\rho\left(P^{m} f\right)\right]^{-1}$, где $f \in C_{0}^{+}$, a $m=0,1, \ldots$, не зависят ни от выбора $f$, ни от выбора $m$. В частности, определение параметра $r$ с помощью (1.9) корректно.

Следствие 5. Ecлu $f, g \in C_{0}^{+}, m o\left(P^{n} f, g\right)\left(P^{n+2} f, g\right)^{-1} \longrightarrow r^{2} n p u$ $n \rightarrow \infty$.

Действительно, пусть $g\left(x_{0}\right)>0$. Согласно условию (IIa), $Q^{j} f\left(x_{0}\right)>0$ при некотором $j \geqslant 1$, а стало быть, найдется такая функция $w \in C_{0}^{+}$, что одновременно $g \geqslant w$ и $Q^{j} f \geqslant w$. Сопоставив теорему 1 , лемму 6 и следствие 4 , получаем утверждение следствия 5 , но пока лишь при $n \rightarrow \infty$ по четным значениям. Это же рассуждение с $f$, замененным на $P f$, охватывает случай нечетных $n$.

Доказ а тельст то п редложения 2. В силу (1.9) радиус сходимости степенного ряда (3.5) с $f \in C_{0}^{+}$равен $r$. Вместе с теоремой Фубини это позволяет утверждать, что ряд $\sum_{n \geqslant 0} t^{2 n} P^{2 n} f$ сходится п.в. на компактном носителе $F_{0}$ функции $f$, коль скоро $0 \leqslant t<r$. 
Так как $\xi\left(F_{0}\right)>0$ (см. условие $(\mathrm{I})$ ), найдется минорантная функция $g \leqslant f$. Следовательно, и ряд $\sum_{n \geqslant 0} t^{2 n} P^{2 n} g$ сходится п.в. на $F_{0}$, если $0 \leqslant t<r$. Отсюда $R \geqslant r$ и для завершения доказательства остается сослаться на предложение 1.

Следующие леммы 7 и 8 играют ключевую роль при доказательстве теоремы 3. В них фиксируются функции $g, v \in C_{0}^{+}$и некоторая неубывающая последовательность натуральных чисел $\left\{n_{i} ; i \geqslant 1\right\}$, для которой $a_{i} \equiv\left(Q^{n_{i}} v, g\right)>0$. Под мерами Радона понимаются меры Радона на $E$, и, как обычно, [1], последовательность мер Радона $\left\{\mu_{n} ; n \geqslant 1\right\}$ называется широко сходящейся к мере Радона $\mu$, если $\mu_{n}(f) \rightarrow \mu(f)$ для всех $f \in C_{0}^{+}$, когда $n \rightarrow \infty$.

Лемма 7. Пусть выполнено условие (III). Если меры Радона $\mu_{i}$, определяемье равенством $\mu_{i}(f)=a_{i}^{-1}\left(Q^{n_{i}} f, g\right), f \in C_{0}^{+}$, широко сходятся $\kappa$ мере Радона $\mu$, то $\mu$ является $r^{2}$-инвариантной относительно $Q$ (т.е. относительно ядра $p_{2}(x, d y)$ ).

Д ок аз а т е л с т в о. Замена $p(x, d y)$ на $r p(x, d y)$ позволяет ограничиться рассмотрением случая $r=1$, что мы и сделаем.

$1^{\circ}$. По следствию $5 b_{i} \equiv\left(Q^{n_{i}} f, g\right)\left(Q^{n_{i}+1} f, g\right)^{-1} \longrightarrow 1$ при $i \rightarrow \infty$, где $f \in C_{0}^{+}$. Поэтому

$$
\begin{aligned}
\mu(f) & =\lim _{i \rightarrow \infty}\left[a_{i}^{-1} b_{i}\left(Q^{n_{i}+1} f, g\right)\right]=\lim _{i \rightarrow \infty} a_{i}^{-1}\left(Q^{n_{i}+1} f, g\right) \\
& =\lim _{i \rightarrow \infty} a_{i}^{-1}\left(Q^{n_{i}} w, g\right)=\mu(w)
\end{aligned}
$$

если $w \in C_{0}^{+}$и $w \leqslant Q f$. Выбрав функции $w_{n} \in C_{0}^{+}, n \geqslant 1$, сходящимися снизу к $Q f$ и заставив $w$ пробегать вдоль $\left\{w_{n}\right\}$, получаем из (5.1) неравенство $\mu Q \leqslant \mu$, означающее субинвариантность $\mu$ относительно $Q$.

Если $E$ компактно, широкая сходимость $\mu_{i}$ к $\mu$ возможна только при равномерной ограниченности указанных мер, и в этом случае широкая сходимость мер совпадает с узкой (в вероятностной терминологии - со слабой) сходимостью. При этом аналогично второму из равенств в (5.1)

$$
\mu(f)=\lim _{i \rightarrow \infty} a_{i}^{-1}\left(Q^{n_{i}+1} f, g\right)=\lim _{i \rightarrow \infty} \mu_{i}(Q f)=\mu(Q f)
$$

для всех $f \in C_{0}^{+}$, на чем и заканчивается рассмотрение случая компактного $E$.

$2^{\circ}$. Займемся случаем некомпактного $E$. Фиксировав $\varepsilon>0$ и задав $l$ и $\varphi$ согласно $(1.10)$, докажем неравенство

$$
\sup _{i \geqslant j}\left\{\frac{\mu_{i}\left(1_{E \backslash F} Q^{l} f\right)}{\mu_{i}(\varphi)}\right\}<\varepsilon
$$

при подходящем выборе числа $j=j(\varepsilon)$ и компакта $F=F(\varepsilon) \subset E$. Пусть $\delta>0,0<t<1$ и $Q^{l} f / H_{t} \varphi<\delta$ вне некоторого компакта $F$ (см. (III)). 
То же рассуждение, что и в начале п. $1^{\circ}$, устанавливает неравенство $\left(Q^{n+1} \varphi_{i}, g\right) \leqslant(1+\delta)\left(Q^{n} \varphi_{i}, g\right)$, где $\varphi_{i}=Q^{n_{i}} \varphi$, при цельх $n \geqslant 0$ и достаточно больших $i$. Поэтому

$$
\begin{aligned}
\left(Q^{n} \varphi_{i}, g\right) & \leqslant(1+\delta)^{n}\left(\varphi_{i}, g\right) \\
\left(H_{t} \varphi_{i}, g\right) & =\sum_{n \geqslant 1} t^{2 n}\left(Q^{n} \varphi_{i}, g\right) \leqslant\left[\sum_{n \geqslant 1} t^{2 n}(1+\delta)^{n}\right]\left(\varphi_{i}, g\right) \\
& \leqslant(1-\beta)^{-1}\left(\varphi_{i}, g\right)
\end{aligned}
$$

коль скоро $\beta \equiv t^{2}(1+\delta)<1$ (далее считаем $\delta>0$ удовлетворяющим последнему неравенству). Таким образом, при достаточно больших $i$

$$
\begin{aligned}
\mu_{i}\left(1_{E \backslash F} Q^{l} f\right) & \leqslant \delta \mu_{i}\left(H_{t} \varphi\right)=\delta a_{i}^{-1}\left(H_{t} Q^{n_{i}} \varphi, g\right)=\delta a_{i}^{-1}\left(H_{t} \varphi_{i}, g\right) \\
& \leqslant \delta a_{i}^{-1}(1-\beta)^{-1}\left(\varphi_{i}, g\right)=\delta(1-\beta)^{-1} \mu_{i}(\varphi) .
\end{aligned}
$$

Уменьшив при необходимости $\delta>0$, из (5.5) выводим (5.2).

Оставшаяся часть доказательства проста. Пусть $0<\varepsilon<1$, а функция $u_{\varepsilon} \in C_{0}^{+}$принимает значения из $[0,1]$ и равна 1 на $F=F(\varepsilon)$. Будем считать $i \geqslant j(\varepsilon)$ достаточно большим. Тогда неравенство $\mu_{i}\left(Q^{n} f\right) \geqslant$ $(1-\varepsilon)^{n} \mu_{i}(f), n \geqslant 1$, устанавливаемое по аналогии с (5.3), вместе с (5.2) дает

$$
\begin{aligned}
\mu_{i}\left(u_{\varepsilon} Q^{l} f\right) & \geqslant \mu_{i}\left[\left(1-1_{E \backslash F}\right) Q^{l} f\right] \geqslant \mu_{i}\left(Q^{l} f\right)-\varepsilon \mu_{i}(\varphi) \\
& \geqslant(1-\varepsilon)^{l} \mu_{i}(f)-\varepsilon \mu_{i}(\varphi) .
\end{aligned}
$$

Отсюда при $i \rightarrow \infty$ следует, что $\mu\left(u_{\varepsilon} Q^{l} f\right) \geqslant(1-\varepsilon)^{l} \mu(f)-\varepsilon \mu(\varphi)$. Заставив теперь $\varepsilon$ пробегать значения вида $n^{-1}, n \geqslant 2$, и выбрав соответствующие компакты $F\left(n^{-1}\right)$ не убывающими с ростом $n$, приходим к неравенству $\mu\left(Q^{l} f\right) \geqslant \mu(f)$. Однако в силу субинвариантности $\mu$ (см. п. $1^{\circ}$ ) его левая часть не превышает правую, так что его части равны друг другу, т.е. $\mu$ инвариантна относительно $Q$.

Лемма 8. Пусть выполнено условие (III). Если меры Радона $\varkappa_{i}$, определяемые равенством $\varkappa_{i}(f)=a_{i}^{-1}\left(P Q^{n_{i}} f, g\right), f \in C_{0}^{+}$, широко сходятся $x$ мере Радона $\varkappa$, то она $r^{2}$-инвариантна относительно $Q$.

Д ок аз а т е ль с т в о сходно с предыдущим. Пусть снова $r=1$. Замена $f$ на $P f$ позволяет сначала по аналогии с (5.1) вывести неравенство $\varkappa(f) \geqslant \varkappa(w)$ для всех $f, w \in C_{0}^{+}$, для которых $w \leqslant Q f$, а затем убедиться в субинвариантности $\varkappa$ относительно $Q$.

Предположим $E$ некомпактным, так как рассмотрение компактного $E$ не требует новшеств. Выберем новую функцию $w \in C_{0}^{+}$с соблюдением условий $Q^{j} \varphi \geqslant w$ и $g \geqslant w$ (ср. п. $1^{\circ}$ предыдущего доказательства). При $n_{i}>j$

$$
\left(Q^{n_{i}} \varphi, g\right) \geqslant\left(Q^{n_{i}-j} w, w\right)=\left\|P^{n_{i}-j} w\right\|^{2} \geqslant c_{1}\left\|P^{n_{i}} g\right\|^{2} \geqslant c_{2}\left(Q^{n-i} \varphi, P g\right)
$$


с некоторыми $c_{1}, c_{2}>0$ (в (5.6) предпоследнее неравенство вытекает из лемм 2 и 6, а последнее - из неравенств, приведенных в конце доказательства леммы 2).

Ввиду (5.6) при достаточно больших $i$ и некотором $c_{3}>0$

$$
\left(H_{t} \varphi_{i}, P g\right) \leqslant(1-\beta)^{-1}\left(\varphi_{i}, P g\right) \leqslant c_{e}\left(Q^{n_{i}} \varphi, g\right)=c_{3}\left(\varphi_{i}, g\right)
$$

где опять $0<\beta<1$ (ср. с (5.4)). Благодаря этому, подобно (5.5) и (5.2) находим

$$
\varkappa_{i}\left(1_{E \backslash F} Q^{l} f\right) \leqslant \delta a_{i}^{-1}\left(H_{t} \varphi_{i}, P g\right) \leqslant \delta a_{i}^{-1} c_{3}\left(\varphi_{i}, g\right)<\varepsilon \mu_{i}(\varphi),
$$

где $\varepsilon>0$ можно выбрать произвольно, $\delta=\delta(\varepsilon)>0$ достаточно мало, а $i$ достаточно велико.

Остается в рассуждениях, завершающих предшествующее доказательство, заменить меры $\mu_{i}$ на $\varkappa_{i}$ и вместо (5.2) применить (5.7).

Д оказат ел в с т о т е о р м ы 3 . Как и при доказательстве лемм 7 и 8 , ограничимся случаем $r=1$. Задав $g, v \in C_{0}^{+}$и воспользовавшись следствием 5, будем работать лишь с настолько большими $n \geqslant 0$, чтобы $b_{n} \equiv\left(Q^{n} v, g\right) \neq 0$. Меры Радона $\nu_{n}$ определим посредством равенства $\nu_{n}(f)=b_{n}^{-1}\left(Q^{n} f, g\right), f \in C_{0}^{+}$.

Для фиксированной функции $f \in C_{0}^{+}$из леммы 6 и следствия 5 получаем

$$
\left(Q^{n} f, g\right) \leqslant a \sum_{0 \leqslant m \leqslant M}\left(Q^{n+m} v, g\right) \leqslant b\left(Q^{n} v, g\right)
$$

при соответствующих $a, b, M>0$. Неравенства (5.8) устанавливают равномерную ограниченность значений этих мер на любом заданном компакте в $E$. Поэтому каждая подпоследовательность, извлеченная из $\left\{\nu_{n}\right\}$, содержит широко сходяшуюся подпоследовательность $\left\{\nu_{n_{i}}\right\}[1]$, причем соответствующая предельная мера Радона $\nu$ инвариантна относительно $Q$ (см. лемму 7) и $\nu(v)=1$. В силу лиувиллевости ядра $p_{2}(x, d y)$ эти свойства меры $\nu$ определяют ее однозначно независимо от конкретного выбора $\left\{\nu_{n_{i}}\right\}$. Следовательно, меры $\left\{\nu_{n}\right\}$ широко сходятся к $\nu$ при $n \rightarrow \infty$. В то же время, если $\eta$ - произвольная мера Радона, инвариантная относительно $Q$, исключая $\eta \equiv 0$, то $\nu=\alpha \eta$ с $\alpha=1 / \eta(v)$.

Итак, величина $\nu_{n}(f)=\left(Q^{n} f, g\right)\left(Q^{n} v, g\right)^{-1}$ стремится к $\eta(f) / \eta(v)$ при $n \rightarrow \infty$, каковы бы ни были $f, g, v \in C_{0}^{+}$. Отсюда (ср. вывод (4.2)) вытекает (1.11) с $r=1$ и $m=0$ при условии, что $n \rightarrow \infty$ по четным значениям, а с помощью следствия 5 это заключение распространяется и на все четные $m$. Мало того, те же рассуждения, но со ссылкой на лемму 8 опять прйводят к (1.11) с $r=1$ и четным $m$ при $n \rightarrow \infty$ по нечетным значениям, т.е. нами получено утверждение теоремы при четном $m$. 
Остается проверить, что

$$
\lim _{n \rightarrow \infty} \frac{\left(P^{2 n+1} f, g\right)}{\left(P^{2 n} f, g\right)}=1
$$

для $f, g \in C_{0}^{+}$. Согласно уже доказанному, при $i=0,1$

$$
\alpha_{i} \equiv \liminf _{n \rightarrow \infty} \frac{\left(P^{2 n+i} f, P g\right)}{\left(P^{2 n+i} f, g\right)} \geqslant \lim _{n \rightarrow \infty} \frac{\left(P^{2 n+i} f, w\right)}{\left(P^{2 n+i} f, g\right)}=\frac{\eta(w)}{\eta(g)}
$$

для всех $w \in C_{0}^{+}$, не превышающих $P g$, так что $\alpha_{i} \geqslant \eta(P g) / \eta(g)$. На основании следствия 5 отсюда получаем

$$
\begin{aligned}
1 & =\lim _{n \rightarrow \infty} \frac{\left(P^{2 n+2} f, g\right)}{\left(P^{2 n} f, g\right)}=\lim _{n \rightarrow \infty}\left[\frac{\left(P^{2 n} f, P g\right)}{\left(P^{2 n} f, g\right)} \cdot \frac{\left(P^{2 n+1} f, P g\right)}{\left(P^{2 n+1} f, g\right)}\right] \\
& \geqslant \alpha_{0} \alpha_{1} \geqslant \frac{\eta(P g)}{\eta(g)}
\end{aligned}
$$

т.е. $\eta P \leqslant \eta$. Поэтому

$$
\eta(g)=\eta\left(P^{2} g\right)=\int P g d(\eta P) \leqslant \int P g d \eta \leqslant \eta(g)
$$

(здесь использована инвариантность $\eta$ относительно $Q=P^{2}$ ). Следовательно, $\eta P=\eta$, и, сочетая это равенство с ранее сказанным, последовательно проверяем утверждения: а) $\alpha_{0}, \alpha_{1} \geqslant 1$; б) последнее из отношений в (5.11) равно 1 ; в) $\alpha_{0}=\alpha_{1}=1$.

При $i=0,1$ определим $\beta_{i}$ подобно $\alpha_{i}$, но с верхним пределом вместо нижнего (см. (5.10)). Если бы хоть одно из $\beta_{i}$ превысило 1 , то в силу утверждений а) или в) и второй из пределов в (5.11) превысил бы 1 , что в соответствии с в) и (5.11) невозможно. Таким образом, $\alpha_{i}=\beta_{i}=1$ при $i=0,1$, и (5.9) доказано.

6. Лиувиллевское свойство итераций ядра. С теоремой 3 связан естественный вопрос: при каких условиях лиувиллевское свойство ядра $p(x, d y)$ влечет аналогичное свойство для $p_{2}(x, d y)$ ? Частичный ответ можно найти в предложении 3 , составляющем основной результат пункта. Поэтому, как в п. 3 и п. 4, будем рассматривать измеримое пространство $(E, \mathscr{B})$ со счетнопорожденной $\sigma$-алгеброй $\mathscr{B}$, на которой определена $\sigma$-конечная мера $\xi \not \equiv 0$. На этом пространстве зададим стохастическое ядро $p(x, d y)$, которое назовем лиувиллевым, если любая $\sigma$-конечная мера, инвариантная относительно него и абсолютно непрерывная относительно $\xi$, совпадает с $\xi$ с точностью до постоянного множителя (понятие топологически лиувиллева ядра в п. 6 не используется).

Введем новое условие:

(IV) при некотором нечетном $m \geqslant 1$ и всех $x \in E$

$$
\operatorname{var}\left[p_{n}(x, \cdot)-p_{n+m}(x, \cdot)\right] \longrightarrow 0,
$$


когда $n \rightarrow \infty$ (мы следуем стандартному определению расстояния по вариации между мерами [8]).

В соответствии, например, с $[8$, с. 385] левая часть (6.1) не возрастает с ростом $n$, а согласно «закону 0 или $2 \gg[16]$ либо предел левой части (6.1) тождественно равен 0 (к этому и сводится условие (IV)), либо верхняя грань его значений равна 2.

Предложение 3. Пусть неразложимое стохастическое ядро $p(x, d y)$ апериодично и симметрично. Если оно является харрисовским или хотя бы лиувиллевым, но удовлетворяющим условию (IV), то ядро $p_{2}(x, d y)$ также лиувиллево.

Д ок а з т е л ь т в о. Если исходное ядро является харрисовским, то, как отмечалось при доказательстве теоремы 2 , существует множество $E_{1} \in \mathscr{B}$, с которым можно связать харрисовское, а потому лиувиллево сужение ядра $p_{2}(x, d y)$, причем $\xi\left(E \backslash E_{1}\right)=0$, и наше предложение становится очевидным.

Пусть теперь исходное ядро является лиувиллевым и подчиняется (IV), и пусть $\sigma$-конечная мера $\mu$ инварианта относительно $p_{2}(x, d y)$ и абсолютно непрерывна относительно $\xi$. Из выполнения соотношений $\infty>\mu(f)=\mu\left(P^{2} f\right)=\mu P(P f)$ для $\mu$-интегрируемых функций $f \geqslant 0$ вытекает $\sigma$-конечность как $\mu P$, так и $\nu=\mu+\mu P$. Равенство $\nu P=\mu P+\mu P^{2}=\nu$ устанавливает инвариантность $\nu$ относительно ядра $p(x, d y)$, вследствие чего, умножив $\mu$ на подходящий скаляр, получаем право считать $\nu=\xi$.

Положив $h=d \mu / d \xi \geqslant 0$, для всех $f$ прежнего вида имеем

$$
\xi(f)=\nu(f)=\mu(f+P f)=\mu(f)+\xi(h \cdot P f)=\mu(f)+\xi(f \cdot P h)=\xi[f(h+P h)] .
$$

Следовательно, $h+P h=1$ п.в. Поэтому можно а) без ограничения общности предположить, что $h \leqslant 1$ всюду, и б) утверждать (см. лемму 5), что $\xi(E \backslash A)=0$, где $A=\left\{x \in E: P^{n} h(x)+P^{n+1} h(x)=1 ; n=0,1,2, \ldots\right\}$. На $A$, очевидно, действуют равенства

$$
P h=P^{2 n+1} h \quad \text { и } \quad P^{2} h=P^{2 n} h, \quad n \geqslant 1,
$$

которые в сочетании с (IV) показывают, что $P^{2 n} h-P^{2 n+1} h \longrightarrow 0$ на $A$ при $n \rightarrow \infty$. В результате $h=P h=1-h$ п.в. или, иными словами, $h=\frac{1}{2}$ п.в. Таким образом, $\mu=\frac{1}{2} \xi$.

\section{СПИСОК ЛИТЕРАТУРЫ}

1. Бурбаки Н. Интегрирование. М.: Наука, 1977,600 с.

2. Нуммелия $Э$. Общие неприводимые цепи Маркова и неотрицательные операторы. М.: Мир, 1989, 207 с. 
3. Lin $M$. Strong ratio limit theorems for mixing Markov operators. - Ann. Inst. H. Poincaré, 1976, v. 12, № 2, p. 181-191.

4. Orey S. Lecture Notes on Limit Theorems for Markov Chain Transition Probabilities. London: Van Nostrand, 1971, 107 p.

5. Revuz D. Markov Chains. Amsterdam: North-Holland, 1975, 336 p.

6. Tweedie $R$. $L$. $R$-theory for Markov chains on a general state space. Part 1. - Ann. Probab., 1974, v. 2, № 5, p. 840-864.

7. Скороход A. В. Топологически возвратные цепи Маркова. Эргодические свойства. - Теория вероятн. и ее примен., 1986, т. 31, в. 4, с. 641-650.

8. ШІирлев А.Н. Вероятность. М.: Наука, 1989, 638 с.

9. IIIур М.Г. Об асимптотическом поведении многошаговых вероятностей перехода. - Литов. матем. сб., 1980, т. 20, № 4, с. 201-207.

10. IIIур $M . \Gamma$. Асимптотические свойства степеней положительных операторов. I. Теория вероятн. и ее примен., 1984, т. 29, в. 4, с. 692-702.

11. IIIур М.Г. К предельным теоремам для отношений, порожденных случайными блужданиями в однородных пространствах. I, II. - Теория вероятн. и ее примен., 1988 , т. 33 , в. 4 , с. $706-719 ; 1989$, т. 34 , в. 3 , с. $516-527$.

12. IIIyp M.Г. К теореме об асимптотической равнораспределенности сверточных степеней симметричных мер на унимодулярной группе. - Матем. заметки, 1996, T. 60 , № 1 , c. $120-126$.

13. Shur $M$. G. Strong ratio limit theorems for self-adjoint transition operators. - Statistics and Control of Stochastic Processes. Ed. by N. V. Krylov et al. New York: Optimization Software, 1985, p. 428-450.

14. Спичер $Ф$. Принципы случайного блуждания. М.: Мир, 1969, 472 с.

15. Иосида К. Функциональный анализ. М.: Мир, 1967, 624 с.

16. Derriennic $Y$. Lois «zero-deux» pour les processus de Markov. - Ann. Inst. H. Poincaré, 1976, v. 12, № 2, p. 111-129. 